\title{
Asymptotics of the Packet Speed and Cost in a Mobile Wireless Network Model
}

\author{
Ioannis Kontoyiannis* ${ }^{\ddagger}$, Stavros Toumpis*, Riccardo Cavallari ${ }^{\dagger}$, and Roberto Verdone ${ }^{\dagger}$ \\ *Department of Informatics, Athens University of Economics and Business, Greece \\ ${ }^{\ddagger}$ Department of Engineering, University of Cambridge, UK \\ ${ }^{\dagger}$ Department of Electrical, Electronic, and Information Engineering, University of Bologna, Italy \\ Email: i.kontoyiannis@eng.cam.ac.uk, toumpis@aueb.gr, \{riccardo.cavallari, roberto.verdone\}@unibo.it
}

\begin{abstract}
An infinite number of nodes move in $\mathbb{R}^{2}$ according to a random waypoint model; a single packet is traveling towards a destination (located at an infinite distance away) using combinations of wireless transmissions and physical transport on the buffers of nodes. In earlier work [1] we defined two performance metrics, namely, the long-term average speed with which the packet travels towards its destination, and the rate with which transmission cost accumulates with distance covered. Explicit expressions were derived for these metrics, under specific ergodicity assumptions. In this paper we give a precise description of the induced Markov process, we show that it is indeed (uniformly) geometrically ergodic, and that the law of large numbers holds for the random variables of interest. In particular, we show that the two performance metrics are well-defined and asymptotically constant with probability one.
\end{abstract}

Index Terms-Delay-tolerant network, geographic routing, mobile wireless network, packet speed, cost.

\section{INTRODUCTION}

Numerous mobile wireless networks have recently been studied, where packets travel towards their destination using both wireless transmissions and physical transport on the buffers of nodes. Examples include satellite [2], vehicular [3], and pocket-switched [4] networks.

Motivated, in part, by these and related applications, in recent work [1], we studied a stochastic/geometric model for such a network, in which an infinite number of nodes move on the infinite plane according to a random waypoint model, and a single packet is traveling towards a destination located at an infinite distance away. In this setting, we defined the packet speed and the packet cost to be the limits (as the packet trajectory length goes to infinity) of the long-term average rates with which distance is covered over time and transmission cost is accumulated over distance covered, respectively. We computed explicit expressions for these limits, but under the provision that the strong law of large numbers (SLLN) holds for a collection of random variable (RV) sequences describing the evolution of the trajectory of the system.

In this work, we develop simple, natural conditions under which we prove that, indeed, the SLLN holds, thus providing a crucial step in the calculation of the performance metrics. As an intermediate result, we clarify the conditions needed for the long-term average rates to converge to their limits as well as the rate with which this occurs, thus illuminating the applicability of our results in practical settings.
In Section II we review the relevant network model [1]; in Section III we recall the approximations and relevant quantities appearing in [1] that will be needed in this work; our main results appear in Section IV; their proofs are given in the Appendix. A unified treatment of the present results together with those in [1] can be found in [5].

\section{NeTWORK MODEL}

Regarding the node mobility model, at $t=0$ we place an infinite number of nodes on $\mathbb{R}^{2}$ according to a Poisson point process (PPP) with density $\lambda$. Then, each node moves along a straight line, with speed $v_{0}$, changing its travel direction at the event times of a Poisson process of rate $r_{0}$. Nodes move independently of each other and select their travel direction independently of past travel directions and according to the uniform distribution. We describe travel directions using the angle $\theta \in[-\pi, \pi)$ they form with the positive $x$-axis.

Regarding the transmission cost, we assume that when a packet gets transmitted from a node $A$ to a node $B$, such that the vector from $A$ to $B$ is $\mathbf{r}$, then the transmission incurs a cost $C(\mathbf{r})$. All transmissions are instantaneous.

Regarding the traffic, we assume a single packet is created, at time $t=0$, at some node, and needs to travel towards a destination that is located at an infinite distance away, in the direction of the positive $x$-axis. The packet travels towards the destination according to some routing rule (RR) that uses both wireless transmissions and extended stays at node buffers.

Observe that the trajectory of the packet is comprised of linear segments, each segment corresponding to either a wireless transmission or a sojourn on the buffer of a node while the node is not changing its travel direction. For this reason, we break the journey of the packet into stages, indexed by $i=1,2, \ldots$, each stage corresponding to either a wireless transmission, in which case we call it a wireless stage (WS), or a sojourn along a straight line segment, in which case we call it a buffering stage (BS).

We describe each stage $i$ in terms of a number of RVs. First, let $\Delta_{i}$ be its duration; note that $\Delta_{i}=0$ if stage $i$ is a WS. Second, let $C_{i}$ be its transmission cost; note that $C_{i}=0$ if stage $i$ is a BS. Third, let $\Theta_{i}$ be the travel direction of the receiver, if stage $i$ is a WS, or the travel direction of the packet holder, if stage $i$ is a BS. Fourth, let $X_{W, i}$ be the change in the $x$-coordinate of the packet due to the wireless transmission; 
if the stage is a BS, then $X_{W, i}=0$. The change $Y_{W, i}$ in the $y$-coordinate of the packet due to the wireless transmission is defined analogously. Likewise, let $X_{B, i}$ be the change in the $x$-coordinate of the packet due to buffering; if stage $i$ is a WS, then $X_{W, i}=0$. Finally, let $X_{i}=X_{B, i}+X_{W, i}$.

We use two metrics to describe the performance of the RR. The first metric is the speed $V_{p}$, defined as the limit of the long-term average speed with which the packet is traveling towards its destination,

$$
V_{p}=\lim _{n \rightarrow \infty} \frac{\sum_{i=1}^{n} X_{i}}{\sum_{i=1}^{n} \Delta_{i}},
$$

provided the limit exists. The second metric is the cost

$$
C_{p}=\lim _{n \rightarrow \infty} \frac{\sum_{i=1}^{n} C_{i}}{\sum_{i=1}^{n} X_{i}},
$$

again whenever the limit exists. In this work, we will specify a RR for which we will show that both $V_{p}$ and $C_{p}$ exist and are constant with probability one.

To specify the RR, first, let the forwarding region (FR) $\mathcal{F}$ be a nonempty, closed, bounded, and convex subset of $\mathbb{R}^{2}$ that contains $\mathbf{0}$. Second, let the potential $U(\phi, \mathbf{r}):[-\pi, \pi) \times$ $\mathcal{F} \rightarrow \mathbb{R}$ be a continuous function that describes the suitability of a node located at $\mathbf{r}$ with respect to the packet holder and traveling in direction $\theta \in[-\pi, \pi)$, for receiving the packet; the larger the potential, the more suitable the node. Note that the current holder's potential is $U(\theta, \mathbf{0})$, where $\theta$ is its direction of travel. For any node $A$, let $\mathcal{F}(A)$ be the FR shifted by its location $\mathbf{r}_{A}$, i.e., $\mathcal{F}(A)=\mathcal{F}+\mathbf{r}_{A}$.

Given $\mathcal{F}$ and $U(\theta, \mathbf{r})$, the RR is simple: the packet constantly aims to be at the node with the largest potential among all nodes in $\mathcal{F}(A)$, where $A$ is its current holder. Therefore, if $A$ has the largest potential, the packet stays at its buffer; if another node $B$ is found with a higher potential (because $A$ changed its travel direction, or $B$ changed its direction, or $B$ entered $\mathcal{F}(A)$ ), then the packet is transmitted to $B$; that transmission may be immediately followed by one or more transmissions.

The following assumptions are introduced for reasons of mathematical convenience.

Assumption 1: If $\left|\theta_{1}\right|>\left|\theta_{2}\right|$, then $U\left(\theta_{2}, \mathbf{r}\right)>U\left(\theta_{1}, \mathbf{r}\right)$ : If the travel direction improves, the potential becomes better.

Assumption 2: If $U\left(\theta_{1}, \mathbf{r}_{1}\right)>U\left(\theta_{2}, \mathbf{r}_{2}\right)$, then for any $\mathbf{r}_{3}$, $U\left(\theta_{1}, \mathbf{r}_{1}-\mathbf{r}_{3}\right)>U\left(\theta_{2}, \mathbf{r}_{2}-\mathbf{r}_{3}\right)$. This means that if a node $A$ is better than a node $B$ according to some node, it will also be better than $B$ according to all other nodes in their neighborhood. This assumption prevents routing loops.

Assumption 3: Let $\mathcal{K}\left(\theta, \theta^{\prime}\right)$ be the subset of the FR where $U\left(\theta^{\prime}, \mathbf{r}\right)>U(\theta, \mathbf{0})$. Therefore, nodes entering $\mathcal{K}\left(\theta, \theta^{\prime}\right)$ with travel direction $\theta^{\prime}$ become eligible to receive the packet. Let $\mathbf{b}\left(s ; \theta, \theta^{\prime}\right)$ be a parametrization of the boundary of $\mathcal{K}\left(\theta, \theta^{\prime}\right)$ with $s \in[0,1]$. We assume that the derivative $\mathbf{b}^{\prime}\left(s ; \theta, \theta^{\prime}\right)$ exists a.e. in $[0,1]$ and there is a constant $M_{b}$ such that, where the derivative exists, $\left|\mathbf{b}^{\prime}\right|\left(s ; \theta, \theta^{\prime}\right)<M_{b}$.

Assumption 4: The value of $U(-\pi, \mathbf{r})$ is equal to a constant $K$ for all $\mathbf{r} \in \mathcal{F}$. Therefore, the direction $\theta=-\pi$ is uniformly the worst, irrespective of the location $\mathbf{r}$ of a candidate neighbor. Note, however, that the behavior of $U(\theta, \mathbf{r})$ as a function of $\theta$ can strongly depend on $\mathbf{r}$, so that 'good' locations can be favored, in terms of the potential assigned to them, as long as nodes at those locations are not traveling in direction $-\pi$.

\section{PReliminary Results}

We begin by recalling two approximations and a number of quantities introduced in [1], where detailed derivations can be found.

The following intuitive approximation is introduced for reasons of mathematical tractability. It introduces errors, however simulations show that these errors are typically quite modest, i.e., on the order of no more than $10 \%$ [1], [5].

Second Order Approximation: When a node $A$ receives a packet from a node $B$, the mobility process is restarted, except that $A$ maintains its position and travel direction and all new nodes creaed in $\mathcal{F}(A) \cap \mathcal{F}(B)$ with potential greater than the potential of $A$ are removed. Also, when the node $A$ carrying the packet changes its travel direction $\theta$ to some $\theta^{\prime}$, the mobility process is restarted, except that $A$ maintains its position and travel direction and all new nodes created in $\mathcal{F}(A)$ whose potential is greater than $\max \left\{U(\theta, \mathbf{0}), U\left(\theta^{\prime}, \mathbf{0}\right)\right\}$ are removed.

First, consider the setting where node $A$, traveling with direction $\theta$, has just received a packet from node $B$, such that the location of $A$ with respect to $B$ is $\mathbf{r}$. Let $\mathcal{G}(\mathbf{r})=$ $\mathcal{F}(A) \cap(\mathcal{F}(B))^{c}$, i.e., the new region the packet discovers upon arriving at node $A$ and where eligible nodes can be found. Let $E(N ; \theta, \mathbf{r})$ be the expected number of nodes in $\mathcal{G}(\mathbf{r})$ with potential larger than the potential of $A$; let $P_{E}(\theta, \mathbf{r})$ be the probability that $\mathcal{G}$ is empty of such nodes; let $g\left(\theta^{\prime}, \mathbf{r}^{\prime} ; \theta, \mathbf{r}\right)$ be the joint density of the location $\mathbf{r}^{\prime}$ and the direction $\theta^{\prime}$ of an eligble node to which the packet is immediately transmitted upon its arrival at $A$. Expressions for these three functions appear in [1], [5].

Secondly, consider the setting where the packet has, at time $t=0$, just started traveling with direction $\theta$ on the buffer of a node $A$. With a slight abuse of notation we define the following rates; explicit expressions for these rates appear in [1], [5].

1) Let $r_{\mathcal{A}}\left(\theta, \theta^{\prime}\right)$ be such that the infinitesimal probability that node $A$ will change its direction from $\theta$ to a direction in $\left[\theta^{\prime}, \theta^{\prime}+d \theta^{\prime}\right]$ and a new sojourn will commence at the same node in the time interval $[0, d t]$ is $r_{\mathcal{A}}\left(\theta, \theta^{\prime}\right) d \theta^{\prime} d t$.

2) Let $r_{\mathcal{B}}\left(\theta, \theta^{\prime}, \mathbf{r}^{\prime}\right)$ be such that the infinitesimal probability that, within the time interval $[0, d t]$, node $A$ will change its travel direction and this will precipitate a transmission to a node $B$ located within a region of infinitesimal area $d A$ centered at $\mathbf{r}^{\prime}$ and traveling with direction in $\left[\theta^{\prime}, \theta+\right.$ $\left.d \theta^{\prime}\right]$ is $r_{\mathcal{B}}\left(\theta, \theta^{\prime}, \mathbf{r}^{\prime}\right) d \theta^{\prime} d A d t$.

3) Let $r_{\mathcal{C}}\left(\theta, \theta^{\prime}, \mathbf{r}^{\prime}\right)$ be such that the infinitesimal probability that, within the time interval $[0, d t]$, a node $B$ located within a region of infinitesimal area $d A$ centered at $\mathbf{r}^{\prime}$ and traveling with some direction $\theta^{\prime \prime}$ will change its 
direction to lie in $\left[\theta^{\prime}, \theta+d \theta^{\prime}\right]$ and will thus become eligible to receive the packet, is $r_{\mathcal{C}}\left(\theta, \theta^{\prime}, \mathbf{r}^{\prime}\right) d \theta^{\prime} d A d t$.

4) Let $r_{\mathcal{D}}\left(\theta, \theta^{\prime}, s\right)$ be such that the infinitesimal probability that within the time interval $[0, d t]$, a node $B$ traveling with direction $\theta^{\prime}$ crosses the boundary $\mathbf{b}\left(s ; \theta, \theta^{\prime}\right)$ in the section $[s, s+d s]$ is $r_{\mathcal{D}}\left(\theta, \theta^{\prime}, s\right) d \theta^{\prime} d s d t$.

5) Let $r(\theta)$ be such that the infinitesimal probability that any of the above events will occur in the time interval $[0, d t]$ is $r(\theta) d t$.

We now introduce our second approximation:

Time Invariance Approximation: If at time $t=0$ the packet arrived at node $A$ and at time $t=t_{0}>0$ the packet is still with $A$ and $A$ has not changed travel direction, the conditional distribution of all future events describing the end of the current stage is the same as for $t_{0}=0$.

Intuitively, as long as node $A$ is carrying the packet, the mobility process of all other nodes is constantly regenerated, so that the probabilities of the various stage-ending events occurring remain fixed and given by the rates defined above.

\section{Performance Metrics}

\section{A. The Markov chain}

We define the state $S_{i}$ associated with each stage $i \geq 1$, by $S_{i} \triangleq\left(\Theta_{i},\left(X_{W, i}, Y_{W, i}\right)\right)$ if stage $i$ is a WS, and by $S_{i} \triangleq$ $\left(\Theta_{i},(0,0)\right)$ if stage $i$ is a BS.

The associated state space in which each $S_{i}$ takes values is $\mathcal{S} \triangleq \mathcal{S}_{B} \cup \mathcal{S}_{W}$, where the buffering state space $\mathcal{S}_{B} \triangleq$ $[-\pi, \pi) \times\{0\}$, and the wireless state space $\mathcal{S}_{W} \triangleq(-\pi, \pi) \times$ $(\mathcal{F}-\{\mathbf{0}\})$.

Observe that, due to the Second Order Approximation, the process $\left\{S_{i}, i=1,2, \ldots\right\}$ forms a Markov chain: If $S_{i}=$ $(\theta, \mathbf{0})$, i.e., stage $i$ is a BS, then at the start of that stage the complete mobility model was restarted, except that the carrier $A$ kept its direction of travel $\theta$ and its FR did not contain nodes with a potential higher than that of $A$, i.e., $U(\theta, \mathbf{0})$. Likewise, if $S_{i}=\left(\theta_{i}, \mathbf{r}\right)$ with $\mathbf{r} \neq \mathbf{0}$, i.e., in stage $i$ the packet was transmitted from a node $B$ to a node $A$ located at $\mathbf{r} \in \mathcal{F}(B)$, then, at the moment $A$ received the packet, the whole mobility model was again restarted, except that $A$ kept its direction of travel $\theta$ and all nodes with potential higher than $U(\theta, \mathbf{r})$ were expunged from $\mathcal{F}(A) \cap \mathcal{F}(B)$. In both cases, the complete information remaining about the network is captured in the current state.

We now define one last rate function. Let $r_{\hat{\mathcal{D}}}\left(\theta, \theta^{\prime}, \mathbf{r}^{\prime}\right)$ be such that the infinitesimal probability that within the time interval $[0, d t]$, a node $B$ located within a region of infinitesimal area $d A$ centered at $\mathbf{r}^{\prime}$ and traveling with direction $\theta^{\prime}$ becomes eligible by crossing $\mathbf{b}\left(s ; \theta, \theta^{\prime}\right)$, for some $s$, is $r_{\hat{\mathcal{D}}}\left(\theta, \theta^{\prime}, \mathbf{r}^{\prime}\right) d \theta^{\prime} d A d t$. A simple expression for $r_{\hat{\mathcal{D}}}\left(\theta, \theta^{\prime}, \mathbf{r}^{\prime}\right)$ can be easily computed from $r_{\mathcal{D}}\left(\theta, \theta^{\prime}, \mathbf{r}^{\prime}\right)$; the details are omitted.

The distribution of the chain $\left\{S_{i}\right\}$ may be described as follows, using the Time Invariance Approximation. We assume that $S_{1}=s \in \mathcal{S}$ is an arbitrary initial state, and for each $i$, given $S_{i}=(\theta, \mathbf{r})$, the chain moves to a state $S_{i+1}=\left(\theta^{\prime}, \mathbf{r}^{\prime}\right)$ according to the following family of conditional distributions:
1) If $\mathbf{r}=\mathbf{r}^{\prime}=\mathbf{0}$, the conditional density of $S_{i+1}$ is

$$
K_{B B}\left(\theta ; \theta^{\prime}\right)=\frac{r_{\mathcal{A}}\left(\theta, \theta^{\prime}\right)}{r(\theta)} .
$$

2) If $\mathbf{r}=\mathbf{0}$ and $\mathbf{r}^{\prime} \neq \mathbf{0}$, the conditional density of $S_{i+1}$ is

$K_{B W}\left(\theta ; \theta^{\prime}, \mathbf{r}^{\prime}\right)=\frac{r_{\mathcal{B}}\left(\theta, \theta^{\prime}, \mathbf{r}^{\prime}\right)+r_{\mathcal{C}}\left(\theta, \theta^{\prime}, \mathbf{r}^{\prime}\right)+r_{\hat{\mathcal{D}}}\left(\theta, \theta^{\prime}, \mathbf{r}^{\prime}\right)}{r(\theta)}$.

3) If $\mathbf{r} \neq \mathbf{0}$ and $\mathbf{r}^{\prime} \neq \mathbf{0}$, the conditional density of $S_{i+1}$ is

$$
K_{W W}\left(\theta, \mathbf{r} ; \theta^{\prime}, \mathbf{r}^{\prime}\right)=g\left(\theta^{\prime}, \mathbf{r}^{\prime} ; \theta, \mathbf{r}\right) .
$$

4) If $\mathbf{r} \neq \mathbf{0}$ and $\mathbf{r}^{\prime}=\mathbf{0}$, the conditional density of $S_{i+1}$ is

$$
K_{W B}\left(\theta, \mathbf{r} ; \theta^{\prime}\right)=\delta\left(\theta^{\prime}-\theta\right) P_{E}(\theta, \mathbf{r}) .
$$

We refer to $K_{B B}\left(\theta ; \theta^{\prime}\right), K_{B W}\left(\theta ; \theta^{\prime}, \mathbf{r}^{\prime}\right), K_{W W}\left(\theta, \mathbf{r} ; \theta^{\prime}, \mathbf{r}^{\prime}\right)$, and $K_{W B}\left(\theta, \mathbf{r} ; \theta^{\prime}\right)$ as kernel functions, since they can be used to fully specify the transition kernel of the chain $\left\{S_{i}\right\}$.

\section{B. Ergodicity}

In this section we establish that the Markov chain $\left\{S_{i}\right\}$ is ergodic, with a unique invariant distribution $\pi$, to which it converges at a geometric rate.

Let $\mathcal{L}_{1}$ denote the Lebesgue measure on $[-\pi, \pi), \mathcal{L}_{2}$ denote the Lebesgue measure on $\mathcal{F}$, and $\delta_{\mathbf{0}}$ be the point mass at point $\mathbf{0}=(0,0) \in \mathbb{R}^{2}$. We write $\psi$ for the measure $\psi=$ $\mathcal{L}_{1} \times \delta_{\mathbf{0}}+\mathcal{L}_{1} \times \mathcal{L}_{2}$, defined on the state space $\mathcal{S}$, equipped with the usual Borel $\sigma$-field. Our first result describes the long-term behavior of the chain $\left\{S_{i}\right\}$, and its consequences are stated in detail after that; see [6] for some relevant background on Markov chains. Theorem 1 is proved in the Appendix.

Theorem 1: The Markov chain is $\psi$-irreducible, aperiodic, and uniformly ergodic on the state space $\mathcal{S}$, with a unique invariant measure $\pi$ to which it converges uniformly geometrically fast. In particular:

1) There are constants $B<\infty$ and $\rho \in(0,1)$ such that, for any initial state $s \in \mathcal{S}$,

$$
\left|P\left(S_{n} \in A \mid S_{1}=s\right)-\pi(A)\right| \leq B \rho^{n},
$$

for all $n \geq 1$ and any (measurable) set $A \subset \mathcal{S}$.

2) For any (measurable) function $F: \mathcal{S} \rightarrow \mathbb{R}$ with $E_{\pi}[|F(S)|]<\infty$, as $n \rightarrow \infty$, with probability one,

$$
\frac{1}{n} \sum_{i=1}^{n} F\left(S_{i}\right) \rightarrow E_{\pi}[F(S)]
$$

for any initial state $s \in \mathcal{S}$.

An important ingredient in the proof of Theorem 1 is the following domination condition, which will be verified in the Appendix. Intuitively, Lemma 1 says that, irrespective of the current state, with probability at least $\epsilon$ the chain will be in a uniformly distributed buffering state after two time steps.

Lemma 1: (Doeblin condition) Let $\mu$ denote the measure $\mathcal{L}_{1} \times \delta_{\mathbf{0}}$ on $\mathcal{S}$. There is an $\epsilon>0$ such that, for any (measurable) $A \subset \mathcal{S}$ and any $s \in \mathcal{S}$, we have:

$$
P\left(S_{i+2} \in A \mid S_{i}=s\right) \geq \epsilon \mu(A) .
$$


Another ingredient of the proof of the $\psi$-irreducibility part of Theorem 1 is provided by the following one-step reachability bound. Lemma 2 is proved in the Appendix.

Lemma 2: Let $\mu^{\prime}$ denote the measure $\mathcal{L}_{1} \times \mathcal{L}_{2}$ on $\mathcal{S}_{W}$. For any (measurable) $A \subset \mathcal{S}_{W}$ with $\mu^{\prime}(A)>0$ there are $-\pi \leq \theta_{1}^{\prime}<\theta_{2}^{\prime}<\pi$ such that,

$$
P\left(S_{i+1} \in A \mid S_{i}=(\theta, \mathbf{0})\right)>0, \quad \text { for all } \theta \in\left(\theta_{1}^{\prime}, \theta_{2}^{\prime}\right) .
$$

The main implications of Theorem 1 for our results are stated in the following corollary, which is proved in the Appendix. In order to state it we need some additional definitions. Given an arbitrary state $S_{1}=s=\left(\theta,\left(x_{W}, y_{W}\right)\right)$ in $\mathcal{S}$, let $\Delta_{1}$ be exponentially distributed with rate $r(\theta)$ if $\left(x_{W}, y_{W}\right)=\mathbf{0}$, and $\Delta_{1}=0$ otherwise. Similarly, for each $i \geq 2$, given $\left(S_{1}, \ldots, S_{i-1}, S_{i}=\left(\theta,\left(x_{W}, y_{W}\right)\right)\right)$ and $\left(\Delta_{1}, \ldots, \Delta_{i-1}\right)$, let $\Delta_{i}$ have the same distribution as $\Delta_{1}$ given $\left(\theta,\left(x_{W}, y_{W}\right)\right)$. Then $\left\{\bar{S}_{i}=\left(\Theta_{i},\left(X_{W, i}, Y_{W, i}\right), \Delta_{i}\right)\right\}$ defines a new Markov chain, on the state space:

$\overline{\mathcal{S}}=([-\pi, \pi) \times\{\mathbf{0}\} \times[0, \infty)) \cup((-\pi, \pi) \times(\mathcal{F}-\{\mathbf{0}\}) \times\{0\})$.

Now suppose $S=\left(\Theta,\left(X_{W}, Y_{W}\right)\right)$ has distribution $\pi$ and let $\Delta$ be defined as before, conditional on $S$. Write $\bar{\pi}$ for the induced joint distribution of $\bar{S}=\left(\Theta,\left(X_{W}, Y_{W}\right), \Delta\right)$ on $\overline{\mathcal{S}}$.

Corollary 1: For any initial state $S_{1}=s, \Delta_{1}=\delta$, the following ergodic theorems hold with probability one,

$$
\begin{aligned}
\lim _{n \rightarrow \infty} \frac{1}{n} \sum_{i=1}^{n} X_{W, i} & =E_{\pi}\left(X_{W}\right), \\
\lim _{n \rightarrow \infty} \frac{1}{n} \sum_{i=1}^{n} C_{i} & =E_{\pi}(C)=E_{\pi}\left(C\left(X_{W}, Y_{W}\right)\right), \\
\lim _{n \rightarrow \infty} \frac{1}{n} \sum_{i=1}^{n} \Delta_{i} & =E_{\bar{\pi}}(\Delta), \\
\lim _{n \rightarrow \infty} \frac{1}{n} \sum_{i=1}^{n} X_{B, i} & =E_{\bar{\pi}}\left(X_{B}\right)=v_{0} E_{\bar{\pi}}(\Delta \cos \Theta),
\end{aligned}
$$

where $\left(\Theta,\left(X_{W}, Y_{W}\right), \Delta\right) \sim \bar{\pi}$ so that $\left(\Theta,\left(X_{W}, Y_{W}\right)\right) \sim \pi$.

As the final step of our analysis, we provide expressions for the performance metrics defined in Section II. The following results are immediate consequences of Corollary 1.

Corollary 2: For any initial state $S_{1}=s, \Delta_{1}=\delta$, the limits defining the performance metrics $V_{p}$ and $C_{p}$ in (1) and (2), respectively, exist with probability one, and are given by:

$$
\begin{aligned}
V_{p} & =\frac{E_{\bar{\pi}}\left(X_{W}+v_{0} \Delta \cos \Theta\right)}{E_{\bar{\pi}}(\Delta)}, \\
C_{p} & =\frac{E_{\bar{\pi}}\left(C\left(X_{W}, Y_{W}\right)\right)}{E_{\bar{\pi}}\left(X_{W}+v_{0} \Delta \cos \Theta\right)},
\end{aligned}
$$

where $\left(\Theta,\left(X_{W}, Y_{W}\right), \Delta\right) \sim \bar{\pi}$.

\section{APPENDIX}

Proof of Lemma 1: It is obvious that it suffices to establish the result of the lemma for events of the form $A=A_{0} \times\{\mathbf{0}\}$, for $A_{0} \subset[-\pi, \pi)$. And by the uniqueness of Carathéodory extension, since the collection of all finite unions of intervals forms an algebra that generates the Borel $\sigma$-algebra of $\mathcal{S}$, it further suffices for $A_{0}$ to only consider closed intervals, $A_{0}=$ $\left[\theta_{1}, \theta_{2}\right]$; see, e.g., [7], [8] for details. So in the rest of the proof we restrict attention to events $A$ of the form $A=\left[\theta_{1}, \theta_{2}\right] \times\{\mathbf{0}\}$.

Also note that, from the expressions for the rates given at [1], it is simple to obtain the following bounds on the transition rates $r_{\mathcal{A}}, r_{\mathcal{B}}, r_{\mathcal{C}}, r_{\mathcal{D}}$, and on $r(\theta)$ :

$$
\begin{aligned}
\frac{r_{0}}{2 \pi} \exp [-\lambda|\mathcal{F}|] & \leq r_{\mathcal{A}}\left(\theta, \theta^{\prime}\right) \leq \frac{r_{0}}{2 \pi} \\
r_{\mathcal{B}}\left(\theta, \theta^{\prime}, \mathbf{r}^{\prime}\right), r_{\mathcal{C}}\left(\theta, \theta^{\prime}, \mathbf{r}^{\prime}\right) & \leq \frac{r_{0} \lambda}{2 \pi} \\
r_{\mathcal{D}}\left(\theta, \theta^{\prime}, s\right) & \leq \frac{M_{b} \lambda v_{0}}{\pi} \\
r(\theta) & \leq r_{0}+r_{0} \lambda|\mathcal{F}|+2 M_{b} \lambda v_{0} .
\end{aligned}
$$

Now, if $s$ is of the form $s=(\theta, \mathbf{0})$ for some $\theta \in[-\pi, \pi)$, then for any $-\pi \leq \theta_{1}<\theta_{2}<\pi$,

$$
P\left(S_{i+1} \in\left[\theta_{1}, \theta_{2}\right] \times\{\mathbf{0}\} \mid S_{i}=(\theta, \mathbf{0})\right)=\int_{\theta_{1}}^{\theta_{2}} \frac{r_{\mathcal{A}}\left(\theta, \theta^{\prime}\right)}{r(\theta)} d \theta,
$$

so that, using the lower bound in (6) and the upper bound in (7), we have that, for some fixed constant $\delta_{1}>0$ :

$$
P\left(S_{i+1} \in\left[\theta_{1}, \theta_{2}\right] \times\{\mathbf{0}\} \mid S_{i}=(\theta, \mathbf{0})\right) \geq \delta_{1}\left(\theta_{2}-\theta_{1}\right) .
$$

Then, using the Markov property and applying (8) twice,

$$
\begin{aligned}
& P\left(S_{i+2} \in\left[\theta_{1}, \theta_{2}\right] \times\{\mathbf{0}\} \mid S_{i}=(\theta, \mathbf{0})\right) \\
& \quad \geq P\left(S_{i+1} \in \mathcal{S}_{B} \mid S_{i}=(\theta, \mathbf{0})\right) \delta_{1}\left(\theta_{2}-\theta_{1}\right) \\
& \quad \geq 2 \pi \delta_{1}^{2}\left(\theta_{2}-\theta_{1}\right) .
\end{aligned}
$$

Similarly, if $s$ is of the form $s=(\theta, \mathbf{r})$ for some $\theta \in[-\pi, \pi)$ and $\mathbf{r} \in \mathcal{F}$, then by the Markov property,

$$
\begin{aligned}
P & \left(S_{i+2} \in\left[\theta_{1}, \theta_{2}\right] \times\{\mathbf{0}\} \mid S_{i}=(\theta, \mathbf{r})\right) \\
& \geq P\left(S_{i+1}=(\theta, \mathbf{0}), S_{i+2} \in\left[\theta_{1}, \theta_{2}\right] \times\{\mathbf{0}\} \mid S_{i}=(\theta, \mathbf{r})\right) \\
& =P_{E}(\theta, \mathbf{r}) \int_{\theta_{1}}^{\theta_{2}} \frac{r_{\mathcal{A}}\left(\theta, \theta^{\prime}\right)}{r(\theta)} d \theta^{\prime} \\
& \geq \delta_{1} P_{E}(\theta, \mathbf{r})\left(\theta_{2}-\theta_{1}\right)=\delta_{1} \exp \{-E(N ; \theta, \mathbf{r})\}\left(\theta_{2}-\theta_{1}\right) \\
& \geq \delta_{1} \exp \{-\lambda|\mathcal{F}|\}\left(\theta_{2}-\theta_{1}\right) .
\end{aligned}
$$

The last inequality holds because, using the definition of $E(N ; \theta, \mathbf{r})$, we clearly have $E(N ; \theta, \mathbf{r}) \leq \lambda|\mathcal{F}|$.

Combining (10) and (11) yields the required result, with $\epsilon=\min \left\{2 \pi \delta_{1}^{2}, \delta_{1} \exp \{-\lambda|\mathcal{F}|\}\right\}$.

Proof of Lemma 2: Since $A$ has positive Lebesgue measure, we can find a rectangle of the form $I=\left[\theta_{1}, \theta_{2}\right] \times\left[x_{1}, x_{2}\right] \times$ $\left[y_{1}, y_{2}\right] \subset \mathcal{S}_{W}$ with a nonempty interior, such that $\mu^{\prime}(A \cap$ $I)>0$. The idea of the main argument here is to show that there is a range of angles $\left(\theta_{1}^{\prime}, \theta_{2}^{\prime}\right)$ such that, when the current packet holder travels with a direction in $\left(\theta_{1}^{\prime}, \theta_{2}^{\prime}\right)$, there is a strictly nonzero probability that there are ineligible nodes in $\left[x_{1}, x_{2}\right] \times\left[y_{1}, y_{2}\right]$ that can become eligible by changing their direction of travel to a better one within the range $\left[\theta_{1}, \theta_{2}\right]$.

Since $U$ is continuous, the image $U(I)$ of $I$ is a closed interval $[a, b]$. And since $I$ has a nonempty interior, we must have $a<b$ by Assumption 1. Also, by Assumptions 1 and 4, 
and noting that $\theta_{1}>-\pi$ in order to have $I \subset \mathcal{S}_{W}$, we must have $b>a>U(-\pi, \mathbf{0})$.

Next, pick some $c, d$ such that $U(-\pi, \mathbf{0})<c<d<$ $\min \{U(0, \mathbf{0}), a\}$, and let $\theta_{1}^{\prime}$ and $\theta_{2}^{\prime}$ be such that $U\left(\theta_{1}^{\prime}, \mathbf{0}\right)=c$ and $U\left(\theta_{2}^{\prime}, \mathbf{0}\right)=d$; such angles are guaranteed to exist by the intermediate value theorem. Also, observe that $U$ is continuous on the compact set $[-\pi, 0] \times\left[x_{1}, x_{2}\right] \times\left[y_{1}, y_{2}\right]$, so it is uniformly continuous there, which implies that there is a $\theta_{B}>-\pi$ with $U(\theta, \mathbf{r})<c$ for all $\theta \in\left[-\pi, \theta_{B}\right]$ and all $\mathbf{r} \in\left[x_{1}, x_{2}\right] \times\left[y_{1}, y_{2}\right]$.

Now take $\left(\theta^{\prime}, \mathbf{r}^{\prime}\right) \in I$ and $\theta \in\left(\theta_{1}^{\prime}, \theta_{2}^{\prime}\right)$ arbitrary. We will bound $r_{\mathcal{C}}\left(\theta, \theta^{\prime}, \mathbf{r}^{\prime}\right)$, given, in Section V-B of [1], by

$$
\begin{aligned}
& r_{\mathcal{C}}\left(\theta, \theta^{\prime}, \mathbf{r}^{\prime}\right)=\frac{\lambda r_{0}}{4 \pi^{2}} \mathbf{1}[\left.U\left(\theta^{\prime}, \mathbf{r}^{\prime}\right)>U(\theta, \mathbf{0})\right] \\
& \times \int_{-\pi}^{\pi} \mathbf{1}\left[U\left(\theta^{\prime \prime}, \mathbf{r}^{\prime}\right)<U(\theta, \mathbf{0})\right] d \theta^{\prime \prime},
\end{aligned}
$$

from below. First note that $U\left(\theta^{\prime}, \mathbf{r}^{\prime}\right)>d$ and $U(\theta, \mathbf{0})<$ $d$, therefore $\mathbf{1}\left[U\left(\theta^{\prime}, \mathbf{r}^{\prime}\right)>U(\theta, \mathbf{0})\right]=1$. Also, we have $U\left(\theta^{\prime \prime}, \mathbf{r}^{\prime}\right)<c<U(\theta, \mathbf{0})$ for all $\theta^{\prime \prime} \in\left[-\pi, \theta_{B}\right]$. Therefore,

$$
r_{\mathcal{C}}\left(\theta, \theta^{\prime}, \mathbf{r}^{\prime}\right) \geq \frac{\lambda r_{0}}{4 \pi^{2}} \int_{-\pi}^{\theta_{B}} d \theta^{\prime \prime}=\frac{\lambda r_{0}}{4 \pi^{2}}\left(\theta_{B}+\pi\right)>0 .
$$

Also recall that $r(\theta)$ is bounded above as in (7).

We are now ready to prove the inequality (3). For any $\theta \in$ $\left(\theta_{1}^{\prime}, \theta_{2}^{\prime}\right)$, where the interval $\left(\theta_{1}^{\prime}, \theta_{2}^{\prime}\right)$ is chosen above,

$$
\begin{gathered}
P\left(S_{i+1} \in A \mid S_{i}=(\theta, \mathbf{0})\right) \geq P\left(S_{i+1} \in A \cap I \mid S_{i}=(\theta, \mathbf{0})\right) \\
\geq \int_{A \cap I} \frac{r_{\mathcal{C}}\left(\theta, \theta^{\prime}, \mathbf{r}^{\prime}\right)}{r(\theta)} d \mu^{\prime}\left(\theta^{\prime}, \mathbf{r}^{\prime}\right)>0 .
\end{gathered}
$$

The last integral is strictly positive because $\mu^{\prime}(A \cap I)$ is nonzero, $r_{\mathcal{C}}\left(\theta, \theta^{\prime}, \mathbf{r}^{\prime}\right)$ is bounded away from zero by (12), and $r(\theta)$ is bounded above by (7).

Proof of Theorem 1: First we will establish the $\psi$ irreducibility and aperiodicity [6] of the chain $\left\{S_{i}\right\}$. In fact, we will show that, for any $n \geq 3$ and any state $s \in \mathcal{S}$, the measure $\psi(\cdot)$ is absolutely continuous with respect to the measure $P\left(S_{i+n} \in \cdot \mid S_{i}=s\right)$. To that end, choose and fix an arbitrary state $s \in \mathcal{S}$ and an arbitrary measurable subset $A$ of $\mathcal{S}$ with $\psi(A)>0$, so that either $\left(\mathcal{L}_{1} \times \delta_{\mathbf{0}}\right)(A)=\mu(A)>0$ or $\left(\mathcal{L}_{1} \times \mathcal{L}_{2}\right)(A)>0$ (or both).

In the first case, Lemma 1 implies that $P\left(S_{i+2} \in A \mid S_{i}=\right.$ $\left.s^{\prime}\right)>0$ for any $s^{\prime}$, which, together with the Markov property, implies that $P\left(S_{i+n} \in A \mid S_{i}=s\right)>0$ for all $n \geq 2$. In the second case, combining Lemma 1 with Lemma 2 applied to $A \cap \mathcal{S}_{W}$ and with the Markov property, we obtain that there are $\theta_{1}^{\prime}<\theta_{2}^{\prime}$ such that,

$$
\begin{aligned}
& P\left(S_{i+3} \in A \mid S_{i}=s\right) \\
& \quad \geq P\left(S_{i+3} \in A, S_{i+2} \in\left(\theta_{1}^{\prime}, \theta_{2}^{\prime}\right) \times\{\mathbf{0}\} \mid S_{i}=s\right) \\
& \quad \geq \epsilon \int_{\theta_{1}^{\prime}}^{\theta_{2}^{\prime}} P\left(S_{i+3} \in A \mid S_{i+2}=(\theta, \mathbf{0})\right) d \theta,
\end{aligned}
$$

where the positivity of the last integral follows again from Lemma 2. Finally, using the Markov property once again, we have that $P\left(S_{i+n} \in A \mid S_{i}=s\right)>0$ for all $n \geq 3$, as required.
Now, $\psi$-irreducibility and aperiodicity, together with the Doeblin bound of Lemma 1, imply [6], [9], that the chain is uniformly ergodic. Specifically, Lemma 1 implies that the state space $\mathcal{S}$ is small, and that the drift condition (V4) of [6] holds with Lyapunov function $V \equiv 1$. Then [6, Theorem 15.0.1] implies that the chain $\left\{S_{i}\right\}$ has a unique invariant (probability) measure $\pi$ to which the distribution of $S_{i}$ converges uniformly, as stated in part 1) of the theorem. In particular, the chain $\left\{S_{i}\right\}$ is Harris recurrent, and [6, Theorem 17.0.1] implies that the strong law of large numbers holds for functions $F \in L_{1}(\pi)$, as stated in part 2) of the theorem.

Proof of Corollary 1: Since $X_{W, i}$ and $C_{i}=C\left(X_{W, i}, Y_{W, i}\right)$ are bounded, and hence $\pi$-integrable, functions of $S_{i}=$ $\left(\Theta_{i},\left(X_{W, i}, Y_{W_{i}}\right)\right)$, the first two results immediately follow from Theorem 1 . For the next two, let $\bar{\psi}$ denote the measure $\bar{\psi}=\mathcal{L}_{1} \times \delta_{\mathbf{0}} \times[0, \infty)+\mathcal{L}_{1} \times \mathcal{L}_{2} \times \delta_{0}$ on $\overline{\mathcal{S}}$. Arguing as in the proof of Theorem 1, it is easy to show that the new chain $\left\{\bar{S}_{i}\right\}$ is $\bar{\psi}$-irreducible and aperiodic, and also uniformly ergodic. Once again, [6, Theorem 17.0.1] implies that the strong law of large numbers holds for $\left\{\bar{S}_{i}\right\}$, and recalling that $X_{B, i}=v_{0} \Delta_{i} \cos \Theta_{i}$, the last two statements of the corollary will follow as soon as we establish that $\Delta$ is $\pi$ integrable. Indeed, since, given $\Theta=\theta, \Delta$ is exponential with rate $r(\theta) \geq r_{0}>0$, we have,

$$
E_{\bar{\pi}}(\Delta)=E_{\pi}\left[E_{\bar{\pi}}(\Delta \mid \Theta)\right]=E_{\pi}\left[\frac{1}{r(\Theta)}\right] \leq \frac{1}{r_{0}}<\infty,
$$

completing the proof.

\section{ACKNOWLEDGMENT}

This work has received funding from the European Union's Horizon 2020 Research and Innovation programme under grant agreement No. 645220 (Road-, Air- and Water-based Future Internet Experimentation - RAWFIE).

\section{REFERENCES}

[1] R. Cavallari, S. Toumpis, and R. Verdone, "Analysis of hybrid geographic/ delay-tolerant routing protocols for wireless mobile networks," in Proc. IEEE INFOCOM, Honolulu, HI, Apr. 2018.

[2] N. Bezirgiannidis, F. Tsapeli, S. Diamantopoulos, and V. Tsaoussidis, "Towards flexibility and accuracy in space dtn communications," in Proceedings of the 8th ACM CHANTS, 2013, pp. 43-48.

[3] P.-C. Cheng, K. C. Lee, M. Gerla, and J. Härri, "GeoDTN+ Nav: geographic DTN routing with navigator prediction for urban vehicular environments," Mobile Networks and Applications, vol. 15, no. 1, pp. 61-82, 2010.

[4] P. Hui, J. Crowcroft, and E. Yoneki, "BUBBLE Rap: Social-based forwarding in delay-tolerant networks," IEEE Transactions on Mobile Computing, vol. 10, no. 11, pp. 1576-1589, 2011.

[5] R. Cavallari, S. Toumpis, R. Verdone, and I. Kontoyiannis, "Analysis of geographic/delay-tolerant routing in mobile wireless networks," Preprint, arXiv:1101.3507, 2018.

[6] S. Meyn and R. Tweedie, Markov Chains and Stochastic Stability, 2nd ed. London: Cambridge University Press, 2009, 1993 edition online: http: //probability.ca/MT/.

[7] H. Royden, Real Analysis. New York: Macmillan, 1988.

[8] D. Williams, Probability with martingales. Cambridge University Press, 1991

[9] I. Kontoyiannis and S. Meyn, "Spectral theory and limit theorems for geometrically ergodic Markov processes," Ann. Appl. Probab., vol. 13, pp. 304-362, February 2003. 